\title{
RADIO SOURCES FROM A 31 GHz SKY SURVEY WITH THE SUNYAEV-ZEL'DOVICH ARRAY
}

\author{
Stephen Muchovej ${ }^{1,2}$, Erik Leitch ${ }^{3}$, John E. Carlstrom ${ }^{3,4}$, Thomas Culverhouse ${ }^{3}$, Chris Greer ${ }^{3}$, David Hawkins ${ }^{1}$, \\ Ryan Hennessy ${ }^{3}$, Marshall Joy ${ }^{5}$, James Lamb ${ }^{1}$, Michael LoH $^{3}$, Daniel P. Marrone ${ }^{3,8}$, Amber Miller ${ }^{6}$, \\ Tony Mroczkowski ${ }^{2,7}$, Clem Pryke ${ }^{3}$, Matthew Sharp ${ }^{3}$, ANd David Woody ${ }^{1}$ \\ ${ }^{1}$ California Institute of Technology, Owens Valley Radio Observatory, Big Pine, CA 93513, USA \\ ${ }^{2}$ Department of Astronomy, Columbia University, New York, NY 10027, USA \\ ${ }^{3}$ Department of Astronomy and Astrophysics, Kavli Institute for Cosmological Physics, University of Chicago, Chicago, IL 60637, USA \\ ${ }^{4}$ Department of Physics, Enrico Fermi Institute, University of Chicago, Chicago IL 60637, USA \\ ${ }^{5}$ Space Sciences-VP62, NASA Marshall Space Flight Center, Huntsville, AL 35812, USA \\ ${ }^{6}$ Columbia Astrophysics Laboratory, Department of Physics, Columbia University, New York, NY, USA \\ ${ }^{7}$ Department of Physics and Astronomy, U Penn, Philadelphia, PA, USA \\ Received 2009 December 11; accepted 2010 April 6; published 2010 May 20
}

\begin{abstract}
We present the first sample of $31 \mathrm{GHz}$ selected sources to flux levels of $1 \mathrm{mJy}$. From late 2005 to mid-2007, the Sunyaev-Zel'dovich Array observed $7.7 \mathrm{deg}^{2}$ of the sky at $31 \mathrm{GHz}$ to a median rms of $0.18 \mathrm{mJy} \mathrm{beam}^{-1}$. We identify 209 sources at greater than $5 \sigma$ significance in the $31 \mathrm{GHz}$ maps, ranging in flux from $0.7 \mathrm{mJy}$ to $200 \mathrm{mJy}$. Archival NVSS data at $1.4 \mathrm{GHz}$ and observations at $5 \mathrm{GHz}$ with the Very Large Array are used to characterize the sources. We determine the maximum-likelihood integrated source count to be $N(>S)=(27.2 \pm 2.5) \mathrm{deg}^{-2} \times\left(S_{\mathrm{mJy}}\right)^{-1.18 \pm 0.12}$ over the flux range $0.7-15 \mathrm{mJy}$. This result is significantly higher than predictions based on $1.4 \mathrm{GHz}$ selected samples, a discrepancy which can be explained by a small shift in the spectral index distribution for faint $1.4 \mathrm{GHz}$ sources. From comparison with previous measurements of sources within the central arcminute of massive clusters, we derive an overdensity of $6.8 \pm 4.4$, relative to field sources.
\end{abstract}

Key words: catalogs - cosmic background radiation - cosmology: observations - radio continuum: general surveys - techniques: interferometric

Online-only material: color figures, machine-readable table

\section{INTRODUCTION}

At the smallest spatial scales, emission from compact extragalactic sources represents the most serious contaminant to measurements of the cosmic microwave background (CMB). The current generation of measurements has reached sensitivities where their power to constrain cosmology rests critically on the ability to characterize the faint-source population. Experiments targeting secondary anisotropies must accurately separate residual source power from the CMB, while surveys for galaxy clusters via their Sunyaev-Zel'dovich (SZ) effect must accurately characterize the source population to understand its implications for cluster detectability. The bulk of these experiments operate at frequencies $\gtrsim 30 \mathrm{GHz}$ where the CMB is brightest, and where the source population is poorly characterized by direct measurement.

Prohibitive integration times have historically limited highresolution surveys for sources at high frequency to small areas, or large flux cutoffs. In recent years, dedicated, multi-year campaigns have produced surveys complete to $\sim 10 \mathrm{mJy}$ at $15 \mathrm{GHz}$, and to $\sim 100 \mathrm{mJy}$ at $20 \mathrm{GHz}$ (Waldram et al. 2003; Massardi et al. 2008; Murphy et al. 2010), but characterization of the compact extragalactic radio source population at $\gtrsim 30 \mathrm{GHz}$ has relied on observations in the $\lesssim 15 \mathrm{GHz}$ range, where the bulk of the sources are brighter, extrapolated to higher frequencies by targeted follow-up campaigns (de Zotti et al. 2005).

In recent years, the development of broadband correlators has made deep surveys of significant areas of sky possible with interferometric arrays operating at frequencies up to $31 \mathrm{GHz}$.

\footnotetext{
8 Jansky Fellow, National Radio Astronomy Observatory.
}

In this paper, we report results of a $7.7 \mathrm{deg}^{2}, 31 \mathrm{GHz}$ sky survey with the Sunyaev-Zel'dovich Array (SZA). Although the primary goal of the SZA was to measure CMB anisotropy and to search for galaxy clusters via their SZ effect, it has also yielded the first catalog of $\sim$ mJy sources selected at $31 \mathrm{GHz}$. This paper focuses on the results of the SZA survey observations as they pertain to the population of high-frequency $(\sim 31 \mathrm{GHz})$ selected compact sources.

Several experiments, such as the Wilkinson Microwave Anisotropy Probe (WMAP; Bennett et al. 2003), DASI (Kovac et al. 2002), CBI (Mason et al. 2003), and the VSA (Cleary et al. 2005), have characterized sources at $31 \mathrm{GHz}$ brighter than $5 \mathrm{mJy}$. The present work extends this characterization to the fainter $31 \mathrm{GHz}$ sources. As these faint sources are at or near the noise level of high-frequency CMB experiments, they represent a serious contaminant which must be carefully modeled and statistically accounted for. The OVRO/BIMA SZ group (Coble et al. 2007) has surveyed $\sim 29 \mathrm{GHz}$ sources associated with massive clusters at the mJy level; the SZA survey allows a comparison of this highly selected population with the bulk properties of field sources at $31 \mathrm{GHz}$. More recently, the CBI collaboration has followed up over $30001.4 \mathrm{GHz}$ selected sources at $31 \mathrm{GHz}$ (Mason et al. 2009) and used these observations to predict the source population at $31 \mathrm{GHz}$. Comparison of this prediction with the SZA measurement at $31 \mathrm{GHz}$ provides a direct test of the assumptions that underlie this extrapolation.

The paper is organized as follows. In Section 2, we present a description of the instrument and of the SZA observations. In Section 3, we describe follow-up observations performed with the Very Large Array (VLA), while Section 4 details the algorithm used to extract source fluxes from the SZA survey 


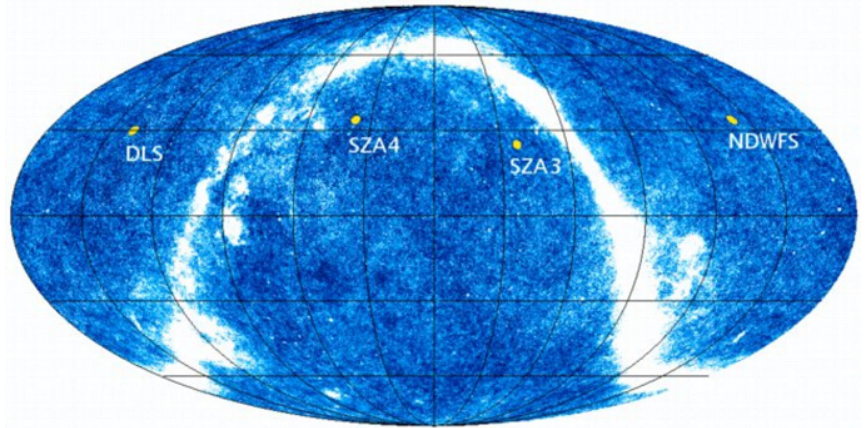

Figure 1. IRAS $100 \mu \mathrm{m}$ dust map with overlay of the SZA field locations. (A color version of this figure is available in the online journal.)

data. The characteristics of the $31 \mathrm{GHz}$ selected sample of sources are presented in Section 5 , followed by a discussion in Section 6. Conclusions are presented in Section 7.

\section{SUNYAEV-ZEL'DOVICH ARRAY OBSERVATIONS}

\subsection{The Sunyaev-Zel'dovich Array}

The SZA is an interferometer designed specifically for detecting and imaging the SZ effect in galaxy clusters, and is located at the Owens Valley Radio Observatory (OVRO). The SZA is equipped with an $8 \mathrm{GHz}$ wideband correlator and sensitive $26-36 \mathrm{GHz}$ and $85-115 \mathrm{GHz}$ receivers. In this paper, we present results only from $26-36 \mathrm{GHz}$ (hereafter $31 \mathrm{GHz}$ ) SZA observations.

The SZA consists of eight $3.5 \mathrm{~m}$ antennas. For the observations presented here, six were arranged in a close-packed configuration (yielding high brightness sensitivity on angular scales typical of clusters of galaxies), and two outlier antennas provided long baselines for sensitivity to compact objects. The SZA can therefore be thought of as two complementary interferometers: one with a typical resolution of a few arcminutes (short antenna separations, or baselines), and the other with a resolution of about 23 arcsec (long baselines). For a more detailed discussion of array layout and corresponding resolution, see Muchovej et al. (2007).

In the limit where sky curvature is negligible over the instrument's field of view, the response of an interferometer on a single baseline, known as a visibility, can be approximated by

$$
V(u, v)=\iint_{-\infty}^{+\infty} A(l, m) I(l, m) e^{-2 \pi i\{u l+v m\}} d l d m
$$

where $u$ and $v$ are the baseline lengths projected onto the sky, $l$ and $m$ are direction cosines measured with respect to the $(u, v)$ axes, $A(l, m)$ is the normalized antenna beam pattern, and $I(l, m)$ is the sky intensity distribution. Equation (1) is a two-dimensional Fourier transform, the inverse of which is the image of the source intensity multiplied by the primary beam pattern, known as a dirty map $I_{D}$ :

$$
\begin{aligned}
I_{D}(l, m) & \equiv A(l, m) I(l, m) \\
& =\iint_{-\infty}^{+\infty} V(u, v) e^{2 \pi i\{u l+v m\}} d u d v
\end{aligned}
$$

In practice, an interferometer measures discrete Fourier modes, and structure in the dirty map is convolved with a function which reflects this incomplete Fourier-space sampling. This function,

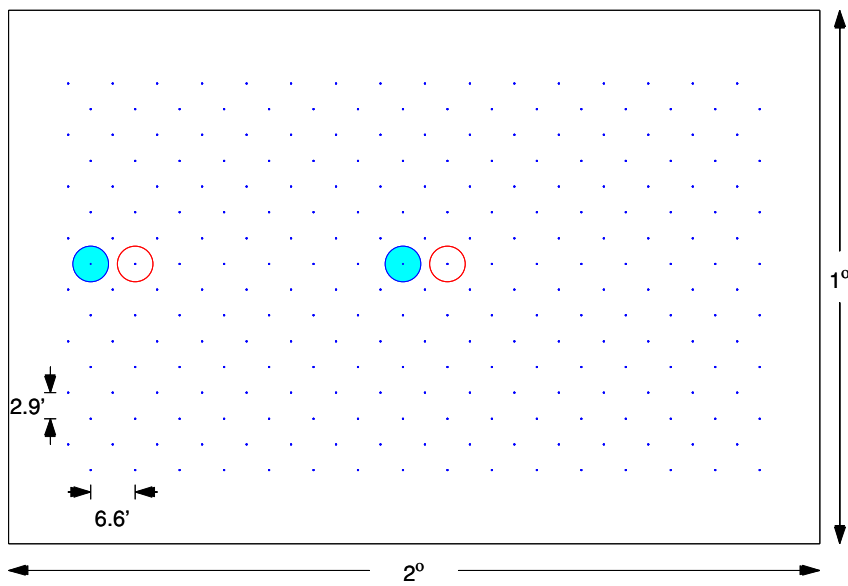

Figure 2. Mosaic pointing locations for a given SZA survey field. The fields are divided into 16 rows of 16 columns, with the pointings in each row separated by 6'.6 and each row offset from each other by $2^{\prime} \cdot 9$. This leads to each field being roughly $2^{\circ} \times 1^{\circ}$ in area. In a single track, the SZA observed four pointings within a given row. For example, pointings in the first and ninth, followed by pointings in the second and tenth columns.

(A color version of this figure is available in the online journal.)

called the synthesized beam, is equivalent to the point-spread function for the interferometer.

It is clear from Equation (2) that the field of view for each dirty map is limited to the size of the primary beam, $A(l, m)$, namely, $\sim 11^{\prime} .0$ (FWHM) for the SZA at band center $(30.938 \mathrm{GHz})$. To image larger areas, we use linear mosaicking to stitch together nearby pointings which cover the region of interest (e.g., Pearson et al. 2003). Note that although large areas of sky can be surveyed in this manner, the resulting mosaics contain no information on size scales larger than that probed by the shortest baseline.

The results presented in this paper are derived from two large SZA projects: one to measure primary CMB anisotropy (Sharp et al. 2010), and the other to survey for galaxy clusters via their SZ effect. Observations were conducted differently for these two projects, as detailed in Section 2.3.

\subsection{Field Selection}

The SZA survey fields were selected to lie far from the plane of the Galaxy and to transit at high elevation at the OVRO site, minimizing atmospheric noise while optimizing the imaging capabilities of the array. Fields were spaced equally in right ascension (R.A.) to permit continuous observation. These constraints led to the selection of four regions ranging in declination from $25^{\circ}$ to $35^{\circ}$. Figure 1 depicts the approximate locations of these four fields overlaid on the IRAS $100 \mu \mathrm{m}$ dust maps (Clegg 1980).

\subsection{Observation Strategy}

\subsubsection{Survey Mode}

Each of the four fields is split into 16 rows of 16 pointings. The pointings are equally spaced by 6.6 along great circles in the R.A. direction, and each row is equally spaced by 2.9 in the decl. direction. Subsequent rows are offset from one another so that that the first pointing in each row is shifted by 3.3 in the R.A. direction relative to the previous row. This means that for a single field we observe an area that spans roughly $2^{\circ}$ in the R.A. direction and $1^{\circ}$ in the decl. direction (see Figure 2). 
Table 1

Survey Observations

\begin{tabular}{|c|c|c|c|c|c|c|c|}
\hline \multirow[t]{2}{*}{ Field Name } & \multicolumn{2}{|c|}{ Field Center (J2000) } & \multicolumn{2}{|c|}{ Calibrators } & \multirow{2}{*}{$\begin{array}{c}\text { Dates } \\
\text { of Observations }\end{array}$} & \multirow{2}{*}{$\begin{array}{l}\text { Integration } \\
\text { Time (hrs) }\end{array}$} & \multirow{2}{*}{$\begin{array}{c}\text { Rows } \\
\text { Covered }\end{array}$} \\
\hline & $\alpha$ & $\delta$ & Bandpass $(\mathrm{Jy})^{\mathrm{a}}$ & $\operatorname{Gain}(\mathrm{Jy})^{\mathrm{a}}$ & & & \\
\hline SZA4 & $02^{\mathrm{h}} 15^{\mathrm{m}} 38^{\mathrm{s}} .3$ & $32^{\circ} 08^{\prime} 21^{\prime \prime}$ & $\mathrm{J} 2253+161(11.6)$ & $\mathrm{J} 0237+288(2.9)$ & $2006 \mathrm{Jul} 11$ to $2007 \mathrm{Jul} 25$ & 687 & 7 \\
\hline DLS & $09^{\mathrm{h}} 19^{\mathrm{m}} 40^{\mathrm{s}} .0$ & $30^{\circ} 01^{\prime} 26^{\prime \prime}$ & $\mathrm{J} 0319+415(11.0)$ & $\mathrm{J} 0854+201(5.4)$ & 2005 Nov 18 to 2007 Jul 6 & 1054 & 14 \\
\hline NDWFS & $14^{\mathrm{h}} 30^{\mathrm{m}} 08^{\mathrm{s}} .0$ & $35^{\circ} 08^{\prime} 34^{\prime \prime}$ & $\mathrm{J} 1229+020(25.3)$ & $\mathrm{J} 1331+305(2.1)$ & 2005 Nov 19 to 2007 Jul 23 & 1000 & 14 \\
\hline SZA3 & $21^{\mathrm{h}} 30^{\mathrm{m}} 07^{\mathrm{s}} \cdot 0$ & $25^{\circ} 01^{\prime} 26^{\prime \prime}$ & $\mathrm{J} 1642+398(5.5)$ & $\mathrm{J} 2139+143(1.4)$ & 2005 Nov 13 to 2007 Jul 25 & 1245 & 16 \\
\hline
\end{tabular}

Note. ${ }^{a}$ Fluxes obtained from $31 \mathrm{GHz}$ SZA observations of sources on 2006 April 16.

For each of the survey fields, data were taken daily in $6 \mathrm{hr}$ tracks. In a single track, we observed two staggered pairs of pointings, all within a single row. These observations were performed in a manner that permits ground subtraction from consecutive pointings in a pair (although ground subtraction was not used in the analysis presented here). Each track results in roughly $1 \mathrm{hr}$ of observation on each of the four pointings, with very nearly the same Fourier sampling for pairs of pointings. A second track is run at a later date, with the order of the pairs reversed, to ensure that the Fourier sampling for all four pointings is comparable. In Figure 2, we show the position of the pointings in each field, and indicate how the pointings were observed in a given track.

For each set of four pointings, this sequence is repeated three times over the span of roughly one year, so that each pointing is observed in six total tracks, translating to roughly $6 \mathrm{hr}$ of observation per pointing over the duration of the survey.

\subsubsection{CMB Anisotropy Observations}

In addition to the survey observations, data were separately taken to measure the anisotropy in the CMB. These consisted of observations of 44 distinct pointings, each separated by $1^{\circ}$, which were not mosaicked, but analyzed individually. Of these 44 pointings, 11 overlap with pointings in the survey fields described above, and the rest are within a $2^{\circ}$ radius of the center of the four survey fields. Where they overlap, the analysis in this paper uses the survey data only. The track structure in the anisotropy observations is similar to that in the survey analysis; see Sharp et al. (2010) for further details.

\subsection{Observations}

Table 1 presents details of the mosaicked SZA survey observations (see Sharp et al. 2010 for the equivalent information on the CMB anisotropy observations). The second and third columns show the approximate pointing center of each 16-row field. We also present the bandpass and gain calibrators in the next two columns, with their fluxes as measured by the SZA. In the fifth column, we give the time range over which observations were taken, with the caveat that observations were not performed every day during that time span. The penultimate column lists the total unflagged integration time for data used in the analysis, and the final column gives the number of rows observed in each field. To ensure uniform coverage of all fields, tracks were repeated when necessary. Note that the full 16 rows were not observed for all fields, due to maintenance operations, instrumental characterization, and Radio Frequency Interference (RFI) monitoring. For the first 8 months of observations, the SZA4 field was used for the dedicated CMB anisotropy measurements described above. As a result, only seven rows in the SZA4 field were completed in survey mode.
The data in the SZA survey correspond to 1493 tracks taken between 2005 November 13 and 2007 July 25. The data in the CMB anisotropy measurements correspond to an additional 414 tracks taken between 2005 November 12 and 2007 October 25. The analysis in this paper refers to the full 1907 tracks taken in both observing modes.

Data for an individual track were calibrated using a suite of MATLAB ${ }^{9}$ routines, which constitute a complete pipeline for flagging, calibrating, and reducing visibility data (Muchovej et al. 2007). Although the data were reduced exactly as described in that paper, data collection differed in a few key ways: four distinct pointings were observed before observing a calibrator, and system temperature measurements were performed every eight minutes. The absolute flux calibration is referenced to Mars, assuming the Rudy (1987) temperature model, and is estimated to be accurate to better than $10 \%$. Typical system temperatures measured throughout the survey were in the range 40-50 K. Flagging of the data as described in Muchovej et al. (2007) resulted in a loss of roughly $15 \%$ of the data. At the end of a single $6 \mathrm{hr}$ track, we achieved a noise level of approximately $1.5 \mathrm{mJy}$ beam $^{-1}$ in each pointing of the short and long baseline maps.

\subsection{Resulting Mosaics}

Once data on all pointings in a given field are reduced, we construct a linear mosaic of the field on a regular grid of 3".3 resolution. This scale is much less than the requirement for Nyquist sampling of the data, $\frac{1}{2 D_{\max }}$, where $D_{\max }$ is the longest baseline, and ensures that the number of pixels over $2^{\circ}$ is a power of 2, convenient for fast inversion of the Fourier data via fast Fourier transform (FFT). The maps are a result of combining the data across our $8 \mathrm{GHz}$ of bandwidth, so that they approximate the sky at the central observing frequency, $30.938 \mathrm{GHz}$. The primary beam is calculated from the Fourier transform of the aperture illumination of each telescope at the central observing frequency, modeled as a Gaussian with a central obscuration corresponding to the secondary mirror.

Unlike the maps of individual pointings, the mosaicked maps are an estimate of the true sky signal at each point in the map; that is, the taper of the primary beam has been divided out. Due to the overlap of neighboring pointings, the effective noise is approximately uniform in the interior of the mosaics, but increases significantly toward the edge of the mosaicked images. We limit the survey area by applying an edge cutoff in our mosaicked maps where the effective noise is $>0.75 \mathrm{mJy}$ beam $^{-1}$ (corresponding roughly to the one-third power point of the beam, given the noise in a single pointing).

\footnotetext{
9 The Mathworks, Version 7.0.4 (R14), http://www.mathworks.com/products/matlab
} 
Table 2

Survey Sensitivity

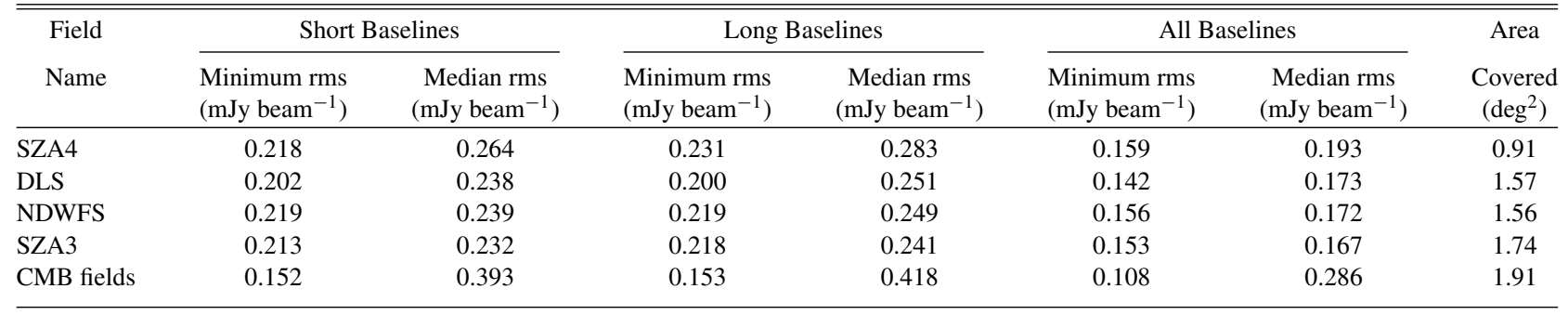

In Table 2, we show the noise properties of the observed fields. We present the minimum and median noise (in $\mathrm{mJy} \mathrm{beam}^{-1}$ ) for mosaic maps made with long baselines only, short baselines only, and with the combination of the two. The median noise is calculated only in the region within which the noise is less than the $0.75 \mathrm{mJy}^{\text {beam }}{ }^{-1}$ cutoff. The last column indicates the total area covered in each field. That the minimum and median pixel noise values are similar is an indication of the uniformity of the coverage in the survey fields. This is not the case in the CMB anisotropy fields as they consisted of discrete pointings which do not overlap on the sky.

\section{VLA OBSERVATIONS}

As described above, the primary goals of the SZA project were a small-scale CMB anisotropy measurement and an SZ survey for clusters of galaxies, both of which require an accurate accounting of foreground emission. Although the long and short baseline data provide some intrinsic ability to discriminate compact objects, as discussed in Section 2, high-sensitivity follow-up observations of the SZA fields were obtained with the VLA ${ }^{10}$ to facilitate source removal.

VLA data at $1.4 \mathrm{GHz}$ are publicly available from the NRAO VLA Sky Survey (NVSS; Condon et al. 1998) for all of the SZA fields, but are limited by the relatively coarse resolution of the NVSS, 45" FWHM, and high detection threshold of $2.5 \mathrm{mJy}$. The finer resolution $\left(5^{\prime \prime}\right)$ and deeper sensitivity (rms of $0.15 \mathrm{mJy}$ ) obtained with the FIRST survey (White et al. 1997) are better suited for this analysis, but data are only available on half of the fields (namely, the Deep Lens Survey (DLS) and NDWFS fields).

To complement the NVSS and FIRST observations, we obtained high-sensitivity VLA observations at $5 \mathrm{GHz}$. Approximately $116 \mathrm{hr}$ of observation were required to cover all four of the SZA fields, taken in four time blocks between 2007 February 24 and 2007 April 15, near the end of the SZA observations. Data were taken at a center frequency of $4.86 \mathrm{GHz}$ with the VLA Darray configuration, by mosaicking 180 pointings in each field. These pointings were arranged in 9 rows of 20 pointings in a hexagonal pattern, equally spaced by 6 arcmin.

The pointings were mosaicked, after CLEANing, using the AIPS package FLATN, with the noise calculated using RMSD. Sources were extracted using the AIPS SAD algorithm, which iteratively removes the brightest point in a mosaic using an elliptical Gaussian model. The rms achieved on the VLA $5 \mathrm{GHz}$ observations was roughly $70 \mu \mathrm{Jy}_{\text {beam }}{ }^{-1}$, resulting in 859 sources from all four fields, down to a $5 \sigma$ depth of $\sim 0.33 \mathrm{mJy}$.

\footnotetext{
10 The Very Large Array is a facility of the National Radio Astronomy Observatory, operated by Associated Universities, Inc., under a cooperative agreement with the National Science Foundation.
}

For the fields observed in CMB anisotropy mode, VLA followup at $8 \mathrm{GHz}$ was obtained. These observations are described in Sharp et al. (2010). In this analysis, we use the VLA data to improve extraction of compact sources from our data as described in Section 4.

\section{SOURCE EXTRACTION FROM THE SZA SURVEY}

\subsection{Overview}

Source identification begins in the image plane, with inspection of the combined (short and long baseline) significance (signal-to-noise ratio $(\mathrm{S} / \mathrm{N})$ ) maps for the brightest pixel with significance greater than 5 . Once we identify the location of a source, we next determine whether the source is extended or unresolved, as seen by the SZA or the VLA, and whether this candidate is a single source, or a collection of nearby sources. Due to the complex sidelobe structure of the synthesized beam (see Section 2), nearby sources must be removed simultaneously from the interferometric data; we therefore fit any additional sources within $45^{\prime \prime}$ of the primary source location, roughly twice the synthesized beam width of the long baseline maps.

Once we have determined all sources near the brightest in the map which are to be removed from the data, as well as their morphology (compact/extended), we solve for source properties by fitting to the multi-pointing visibility data. For computational expediency, we describe the sources as functions with analytic Fourier transforms (see Section 4.2). The best-fit models are removed from the Fourier data, and the mosaics are regenerated. This process is repeated iteratively until there are no sources brighter than $5 \sigma$ in the significance maps.

\subsection{Models}

When fitting sources in the mosaicked maps, we model the intensity distribution of an unresolved source as a delta function,

$$
I(v, \vec{x})=I_{o}\left(\frac{v}{v_{o}}\right)^{-\alpha} \delta\left(\vec{x}-\vec{x}_{o}\right),
$$

where $v$ is the observation frequency, $I_{o}$ is the intensity at frequency $v_{o}$, defined for the SZA as the center frequency of observations, and $\alpha$ is the spectral index.

We model any extended source as a two-dimensional Gaussian, i.e., up to a normalization,

$$
I(v) \propto I_{o}\left(\frac{v}{v_{o}}\right)^{-\alpha} e^{-\frac{\left(l^{\prime}-l_{c}^{\prime}\right)^{2}}{2 \sigma l^{2}}} e^{-\frac{\left(m^{\prime}-m_{c}^{\prime}\right)^{2}}{2 \sigma_{m}^{2}}},
$$

where $l_{c}$ and $m_{c}$ are the coordinates of the centroid, $\theta$ is the orientation angle, and $\sigma_{l}$ and $\sigma_{m}$ are the FWHM of the semimajor and semiminor axes, respectively. In Equation (4), the primed 
coordinate system $\left(l^{\prime}, m^{\prime}\right)$ is related to the unprimed coordinate $(l, m)$ via a rotation by the orientation angle $\theta$.

This model has an analytic Fourier transform provided we can take the primary beam of the SZA to be constant over the extent of the source, i.e., that the source is small compared to the primary beam. In practice, extended models are fitted only to pointings within $6^{\prime}$ of the source, well within the FWHM of the primary beam. For a source $1^{\prime}$ in extent, a factor of $\sim 3$ larger than the most extended source in our data set, this assumption leads to an error in the fitted flux that is below $5 \%$.

\subsection{Unresolved versus Extended Sources}

Sources in the SZA maps are cross-checked against the VLA $5 \mathrm{GHz}$ catalog, and a source is determined to have a counterpart at $5 \mathrm{GHz}$ if a source exists within $8^{\prime \prime}$ of the SZA location, a small fraction of the highest resolution element of the SZA. If a counterpart is found, we consider the source extended if its size at $5 \mathrm{GHz}$ is greater than $22^{\prime \prime} .5$ (the SZA long baseline synthesized beam FWHM). In the rare event that the source has no $5 \mathrm{GHz}$ counterpart (due presumably to source variability or resolution effects when observing extended sources), we check within $8^{\prime \prime}$ of the SZA location in the $1.4 \mathrm{GHz}$ NVSS or FIRST catalogs. When no counterpart is found in any of the catalogs $(\sim 3 \%$ of sources), we compare the flux at that location in the short and long baseline SZA maps to determine if it is extended. If the fluxes are consistent with each other the source is classified as unresolved.

\subsection{Source Location Determination}

To minimize the number of degrees of freedom in each source fit, we fix the location of SZA sources with VLA counterparts to the VLA positions. We have verified that the locations of the VLA counterparts agree with locations fitted to the SZA data to within $3^{\prime \prime}$, and indicate no systematic pointing offsets.

Locations are not fixed for sources determined to be extended, and for very bright sources at $31 \mathrm{GHz}$. The dynamic range (ratio of the fitted source flux to the post-fit residuals) in the mosaicked maps is approximately 35 , limited by the long-term pointing accuracy of the instrument and uncertainties in the primary beam. For sources brighter than $15 \mathrm{mJy}$, however, we can fit the locations accurately in the individual pointings, resulting in an overall dynamic range in the mosaics of 70 , or a factor of 2 improvement. We have verified that agreement in the fitted source location between pointings is at the arcsecond level.

\subsection{Spectral Index Fitting}

For source brighter than $\sim 3.5 \mathrm{mJy}$, the SZA's large bandwidth allows for the simultaneous determination of the spectral index across $8 \mathrm{GHz}$. For fainter sources, however, we cannot meaningfully constrain the spectral index of the source from the SZA data alone. To reduce the number of degrees of freedom for these sources, we use the VLA $5 \mathrm{GHz}$ observations (where available) as a second frequency, fixing the spectral index to

$$
\alpha=-\ln \left(I_{o} / I_{5 \mathrm{GHz}}\right) / \ln \left(v_{o} / 5 \mathrm{GHz}\right)
$$

in Equation (3). Note that these spectral indices are used only to reduce the residuals in the maps when extracting sources. Analysis of the spectral index distribution of these sources by comparison with low-frequency data is presented in Section 5.3.

\section{5. $31 \mathrm{GHz}$ POPULATION CHARACTERISTICS}

\subsection{Source Sample}

Following the procedure outlined above, we identified 209 sources at $31 \mathrm{GHz}$ with $\mathrm{S} / \mathrm{N}$ greater than 5 . From this sample, 40 of the sources were determined to be extended in the VLA data, yet none were seen to be resolved by the SZA (the FWHMs of the fit Gaussians are all smaller than 22.'5), suggesting that the high-frequency emission is dominated by a compact core. In Table 3, we present the full list of sources. The brightest source we detect has a flux of $204 \mathrm{mJy}$. Counterparts were found in the VLA $5 \mathrm{GHz}$ catalog for 162 of the 171 sources with $5 \mathrm{GHz}$ coverage, and $1.4 \mathrm{GHz}$ NVSS counterparts were found for 157 of the 209 sources. All $1.4 \mathrm{GHz}$ sources in the regions for which we have $5 \mathrm{GHz}$ coverage were also found in the $5 \mathrm{GHz}$ data. VLA $8 \mathrm{GHz}$ coverage is available in the region for which we do not have $5 \mathrm{GHz}$ coverage, and all 38 sources detected at $31 \mathrm{GHz}$ in those regions have $8 \mathrm{GHz}$ counterparts (Sharp et al. 2010). Of the 162 sources with $5 \mathrm{GHz}$ counterparts, 34 were found to have higher fluxes at $31 \mathrm{GHz}$ than at $5 \mathrm{GHz}$. In addition, 8 of the 157 sources with $1.4 \mathrm{GHz}$ counterparts were found to be inverted.

\subsection{Source Count}

The differential source count per unit area as a function of flux, $d N / d S$, is typically described by a power law, i.e.,

$$
\frac{d N}{d S}=N_{0}\left(\frac{S}{S_{0}}\right)^{-\gamma},
$$

where $N_{0}$ is a normalization parameter per unit area and flux, $S$ is the source flux, and $\gamma$ is the power-law index. In the analysis, we take $S_{0}=1 \mathrm{mJy}$ and express the normalization as a number of sources per square degree. As models of radio source populations suggest a break in the count near $15 \mathrm{mJy}$ (de Zotti et al. 2005), we fit a power law only to sources below this limit, excluding seven sources from the previous list. Subsequent analysis of the source count is performed on 202 sources.

\subsubsection{Power-law Index, $\gamma$}

Given a set of $N_{S}$ observed source fluxes $\left\{S_{i}\right\}$, we can solve for the underlying population parameters by maximizing the likelihood of the data set,

$$
\mathcal{L}=\prod_{i}^{N_{S}} p\left(S_{i} \mid N_{0}, \gamma, \sigma_{i}\right),
$$

where $S_{i}$ is the observed flux of the source and $\sigma_{i}$ is the map noise at the source location. In the presence of noise, an observed source flux $S$ is the combination of a "true" flux $S_{t}$ and noise $N=S-S_{t}$. The probability in Equation (7) can therefore be written as an integral over all possible pairs of $S_{t}$ and $N$ that will produce an observed flux $S_{i}$ :

$$
\begin{aligned}
p\left(S_{i} \mid N_{0}, \gamma, \sigma_{i}\right) & \propto \int_{0}^{\infty} p_{S}\left(S_{t} \mid N_{0}, \gamma\right) p_{N}\left(S_{i}-S_{t} \mid \sigma_{i}\right) d S_{t} \\
& \propto \int_{0}^{\infty} N_{0} S_{t}^{-\gamma} e^{-\left(S_{i}-S_{t}\right)^{2} / 2 \sigma_{i}^{2}} d S_{t},
\end{aligned}
$$

up to a constant of proportionality (e.g., Murdoch et al. 1973). It is clear that when $p\left(S_{i} \mid N_{0}, \gamma, \sigma_{i}\right)$ is normalized, $N_{0}$ drops out 
Table 3

SZA $30 \mathrm{GHz}$ Sources

\begin{tabular}{|c|c|c|c|c|c|c|}
\hline $\begin{array}{c}\alpha \\
(\mathrm{J} 2000)\end{array}$ & $\begin{array}{c}\delta \\
(\mathrm{J} 2000)\end{array}$ & $\begin{array}{c}S_{t 31 \mathrm{GHz}}^{\mathrm{a}} \\
(\mathrm{mJy})\end{array}$ & $\begin{array}{c}S_{31 \mathrm{GHz}}{ }^{\mathrm{b}} \\
(\mathrm{mJy})\end{array}$ & $\mathrm{S} / \mathrm{N}^{\mathrm{c}}$ & $\begin{array}{c}S_{5 \mathrm{GHz}}{ }^{\mathrm{d}} \\
(\mathrm{mJy})\end{array}$ & $\begin{array}{c}S_{1.4 \mathrm{GHz}}{ }^{\mathrm{e}} \\
(\mathrm{mJy})\end{array}$ \\
\hline $02: 11: 31.56$ & $33: 23: 02.49$ & $4.22_{-0.30}^{+0.28}$ & 4.26 & 14.80 & $\ldots$ & $<3.5$ \\
\hline $02: 11: 40.54$ & $33: 04: 17.31$ & $2.59_{-032}^{+0.30}$ & 2.67 & 8.56 & $\ldots$ & 21.4 \\
\hline $02: 11: 43.10$ & $33: 31: 39.73$ & $4.27_{-0.23}^{+0.21}$ & 4.29 & 19.54 & $\ldots$ & 12.6 \\
\hline $02: 11: 48.05$ & $32: 09: 32.41$ & $1.20_{-0.28}^{+0.26}$ & 1.32 & 5.05 & $\ldots$ & $<3.5$ \\
\hline $02: 12: 43.34$ & $31: 58: 56.93$ & $0.96_{-0.18}^{+0.16}$ & 1.02 & 5.76 & 4.3 & 9.6 \\
\hline $02: 12: 56.83$ & $32: 02: 22.54$ & $1.34_{-0.17}^{+0.16}$ & 1.39 & 8.41 & 2.7 & 5.7 \\
\hline $02: 13: 31.80$ & $31: 58: 08.46$ & $1.10_{-0.17}^{+0.16}$ & 1.16 & 6.84 & 0.4 & $<3.5$ \\
\hline $02: 13: 38.33$ & $31: 58: 48.36$ & $2.12_{-0.17}^{+0.17}$ & 2.15 & 23.64 & 7.6 & $<3.5$ \\
\hline $02: 13: 38.65$ & $31: 59: 07.72$ & $2.67_{-0.17}^{+0.17}$ & 2.69 & 21.09 & 2.2 & $62.8^{f}$ \\
\hline $02: 13: 39.17$ & $31: 59: 27.36$ & $2.37_{-0.18}^{+0.16}$ & 2.39 & 23.85 & 11.4 & $62.8^{f}$ \\
\hline
\end{tabular}

Notes.

${ }^{a}$ Flux corrected for boosting (i.e., $S_{t}$ in Equation (13)). Errors do not reflect uncertainties in the absolute flux calibration.

b Best-fit flux, not accounting for boosting (i.e., $S$ in Equation (13)).

${ }^{c}$ Significance of detection in the composite mosaics.

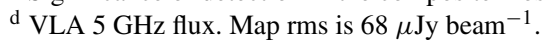

e $1.4 \mathrm{GHz}$ Flux from NVSS catalog.

${ }^{\mathrm{f}}$ Single source in NVSS catalog. $31 \mathrm{GHz}$ fluxes were combined in the $1.4 / 31 \mathrm{GHz}$ spectral index analysis.

(This table is available in its entirety in a machine-readable form in the online journal. A portion is shown here for guidance regarding its form and content.)

of the equation. Thus, this method can only be used to calculate the power-law index $\gamma$; the relative frequency of sources of different flux is related only to the shape parameter $\gamma$, and is independent of the total number of sources. In the noiseless case, it can be trivially shown that the resulting estimator for $\gamma$ meets the Fischer-Neyman criterion for a sufficient statistic, i.e., that it utilizes all the information about the shape parameter contained in the data set (e.g., Crawford et al. 1970).

In the case of nonuniform noise, the above expression leads to a normalized likelihood that depends in a complicated way on the relative areas at different noise levels in the survey. However, because our sources are selected by $\mathrm{S} / \mathrm{N}$ and not flux, it is convenient to transform directly into the $\mathrm{S} / \mathrm{N}$ basis, leading to an expression for the likelihood that is independent of the noise, and therefore of the relative areas at different noise levels:

$$
p\left(s_{i} \mid \gamma\right) \propto \int_{0}^{\infty} s_{t}-\gamma e^{-\left(s_{i}-s_{t}\right)^{2} / 2} d s_{t},
$$

where $s \equiv S / \sigma$. We note in passing that while the kernel of Equation (9) formally diverges as $s_{t} \rightarrow 0$, this is merely an artifact of the implicit assumption that the single-source hypothesis dominates the probability, i.e., that we are considering only sources well above the confusion limit. This assumption is consistent with the results quoted in Section 5.2.3, which indicate that the weakest source flux included in the analysis is approximately 14 times the confusion limit. In practice, we truncate Equation (9) at an $s_{t}$ which corresponds to a flux well above the confusion limit at the lowest map noise, and below which the kernel contributes negligibly to the integral.

We estimate the uncertainty in the calculated value of $\gamma$ by using a quadratic approximation of the likelihood about its maximum. We have verified through extensive simulation that this method produces an unbiased estimate of the power-law parameter $\gamma$ and its associated uncertainty $\sigma_{\gamma}$.

\subsubsection{Normalization, $N_{0}$}

We can invoke Bayes' theorem to write the likelihood of a set of parameters $\left\{N_{0}, \gamma\right\}$ given the data $D$ as

$$
\begin{aligned}
p\left(N_{0}, \gamma \mid D\right) & \propto p\left(D \mid N_{0}, \gamma\right) p\left(N_{0}, \gamma\right) \\
& \propto p\left(D \mid N_{0}, \gamma\right) p\left(N_{0}\right) p(\gamma) .
\end{aligned}
$$

Assuming a uniform prior for $N_{0}, p\left(N_{0}\right)=$ const, and integrating over $\gamma$, we have

$$
p\left(N_{0} \mid D\right) \propto \int p\left(D \mid N_{0}, \gamma\right) p(\gamma) d \gamma .
$$

The distribution $p(\gamma)$ is just the likelihood derived in the last section, or for computational convenience, its Gaussian approximation. The probability of the data given $N_{0}$ and $\gamma$ is the Poisson probability of the observed number of sources $N_{S}$, given the expected number of sources $\lambda\left(N_{0}, \gamma\right)$,

$$
p\left(D \mid N_{0}, \gamma\right) \propto \frac{e^{-\lambda} \lambda^{N_{S}}}{N_{S} !},
$$

where $\lambda\left(N_{0}, \gamma\right)$ is derived by integrating $d N / d S$ over the noise mask of the SZA survey (given in Table 4). The maximumlikelihood estimate of the normalization is then just the value of $N_{0}$ that maximizes $p\left(N_{0} \mid D\right)$.

\subsubsection{Results}

Placing a significance cutoff on the source detections at $5 \sigma$, the source count follows a power law with $\gamma=2.18 \pm 0.12$, and a normalization at $1 \mathrm{mJy}$ of $N_{0}=32.1 \pm 3.0 \mathrm{deg}^{-2} \mathrm{mJy}^{-1}$ (in the 0.7-15 mJy range). Integrating Equation (6) with these parameters yields an integrated source count of

$$
N(>S)=(27.2 \pm 2.5) \mathrm{deg}^{-2} \times\left(S_{\mathrm{mJy}}\right)^{-1.18 \pm 0.12} .
$$

As discussed in Section 2.5, the effective noise in the interior of the mosaicked maps is nearly uniform, but increases rapidly 
Table 4

SZA Area Coverage

\begin{tabular}{lc}
\hline \hline $\begin{array}{c}\text { Noise Value } \\
(\mathrm{mJy})\end{array}$ & $\begin{array}{c}\sum \text { Area } \\
\left(\mathrm{deg}^{2}\right)\end{array}$ \\
\hline 0.11 & 0.000 \\
0.12 & 0.014 \\
0.13 & 0.037 \\
0.14 & 0.080 \\
0.15 & 0.219 \\
0.16 & 0.638 \\
0.17 & 2.578 \\
0.18 & 3.629 \\
0.19 & 4.012 \\
0.20 & 4.281 \\
0.21 & 4.504 \\
0.22 & 4.697 \\
0.23 & 4.868 \\
0.24 & 5.023 \\
0.26 & 5.323 \\
0.28 & 5.554 \\
0.30 & 5.758 \\
0.32 & 5.940 \\
0.34 & 6.106 \\
0.36 & 6.257 \\
0.38 & 6.396 \\
0.40 & 6.525 \\
0.45 & 6.807 \\
0.50 & 7.036 \\
0.55 & 7.226 \\
0.60 & 7.385 \\
0.65 & 7.514 \\
0.70 & 7.613 \\
0.75 & 7.684 \\
\hline
\end{tabular}

toward the edges. Because of this nonuniformity, the $5 \sigma$ significance threshold used to select sources independent of noise level does not translate simply into a uniform completeness limit across the full area of the survey. From the noise distribution, the sample is expected to be $98 \%$ complete above $1.4 \mathrm{mJy}$ over a $4.3 \mathrm{deg}^{2}$ area, but $98 \%$ complete above $5 \mathrm{mJy}$ over the full $7.7 \mathrm{deg}^{2}$ area. Here, we define the $98 \%$ completeness limit as the flux above which the probability of missing a source due to noise fluctuations is $<2 \%$.

\subsection{Spectral Index Distribution}

We calculate spectral indices for all sources in the sample (including those brighter than $15 \mathrm{mJy}$ ) with counterparts in the $5 \mathrm{GHz}$ or $1.4 \mathrm{GHz}$ VLA catalogs assuming a power law, where the spectral index $\alpha$ is defined as in Equation (3) $\left(S_{v} \propto v^{-\alpha}\right)$. For each source, we construct the probability distribution for the spectral index by sampling from the flux distributions at each frequency. (Note that we do not use the $30 \mathrm{GHz}$ spectral index calculated over the SZA's $8 \mathrm{GHz}$ bandwidth, as a power law is not, in general, an accurate representation of the source spectrum over decades of frequency.) In the presence of noise, the conditional probability of the "true" $31 \mathrm{GHz}$ flux $S_{t}$, given an observed flux $S$ is

$$
p\left(S_{t} \mid S, \gamma, \sigma\right) \propto p_{S}\left(S_{t} \mid \gamma\right) p_{N}\left(S-S_{t} \mid \sigma\right)
$$

which is just the kernel of Equation (8). For $\gamma>0, p_{S}\left(S_{t} \mid \gamma\right)$ increases as $S_{t} \rightarrow 0$, and the observed flux $S$ is therefore generally "boosted" by the presence of noise. Marginalizing this kernel over $\gamma$ using the likelihood derived in Section 5.2.1 yields the distribution of $S_{t}$. For the VLA fluxes, no correction
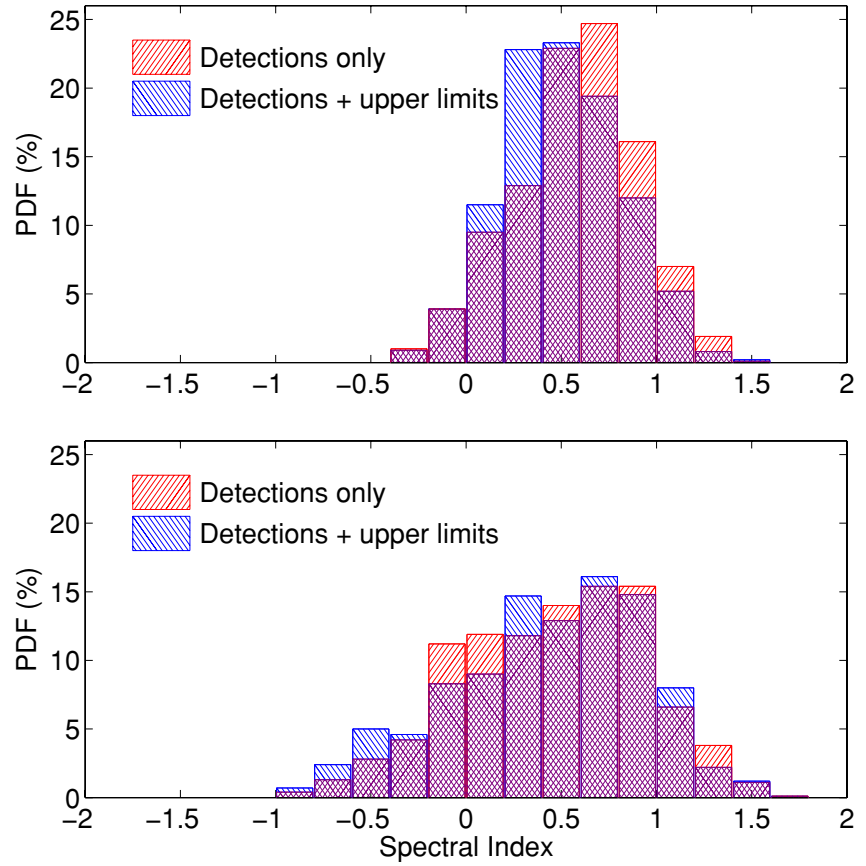

Figure 3. Top: spectral index distribution for $31 \mathrm{GHz}$ selected sources detected with the SZA relative to their $1.4 \mathrm{GHz}$ flux seen by NVSS, where $S_{v} \propto v^{-\alpha}$. Red histograms denote sources with identified counterparts while blue histograms include upper limits for undetected sources, assuming the NVSS completeness limit of $3.5 \mathrm{mJy}$. Bottom: same histograms but for counterparts found in the $5 \mathrm{GHz}$ VLA follow-up data, with a limiting flux of $0.33 \mathrm{mJy}$ at $5 \mathrm{GHz}$.

(A color version of this figure is available in the online journal.)

for boosting has been made. This process is repeated for each source, and the resulting probabilities are co-added to form the spectral index distribution for all sources with counterparts.

By correlating with the NVSS catalog, as described in Section 4.3, we find eight instances where the higher resolution of the SZA and VLA $5 \mathrm{GHz}$ data, compared to NVSS, results in the detection of two sources associated with a single NVSS source. When calculating $1.4 / 31 \mathrm{GHz}$ spectral indices, we treat these eight pairs as a single source. As a result, we calculate 1.4/ $31 \mathrm{GHz}$ for 150 out of 201 sources, shown in the top panel of Figure 3. The distribution peaks at a spectral index of $\alpha \sim 0.7$, consistent with standard synchrotron emission, as expected for most sources bright at $1.4 \mathrm{GHz}$. We note that of the sources with $1.4 \mathrm{GHz}$ counterparts, approximately $5 \%$ are inverted, i.e., have $\alpha<0$. Accounting for sources undetected at $1.4 \mathrm{GHz}$ will likely increase this fraction. For these sources, we use the NVSS completeness limit $(\sim 3.5 \mathrm{mJy})$ to construct upper limits for their spectral indices. Figure 3 illustrates the shift toward more inverted spectral indices when these limits are included.

We repeat the same calculation with the 162 sources with $5 \mathrm{GHz}$ counterparts, noting that only 171 of the 209 sources are covered by the $5 \mathrm{GHz}$ follow-up. The resulting spectral index distribution for these 162 sources is shown in the bottom panel of Figure 3. The distribution peaks at a value of $\alpha \sim 0.8$. Of these 162 sources, we find that $14 \%$ are inverted. Incorporating limits $\left(S_{5 \mathrm{GHz}} \sim 0.33 \mathrm{mJy}\right)$ for nine sources which were not detected at $5 \mathrm{GHz}$ has a minimal effect on the distribution, shifting it marginally toward more inverted sources. Note that this analysis relies on data taken at three different epochs, and takes no account of source variability.

We stress that the intrinsic spectral index distribution of this population of sources can only be inferred from the observed 

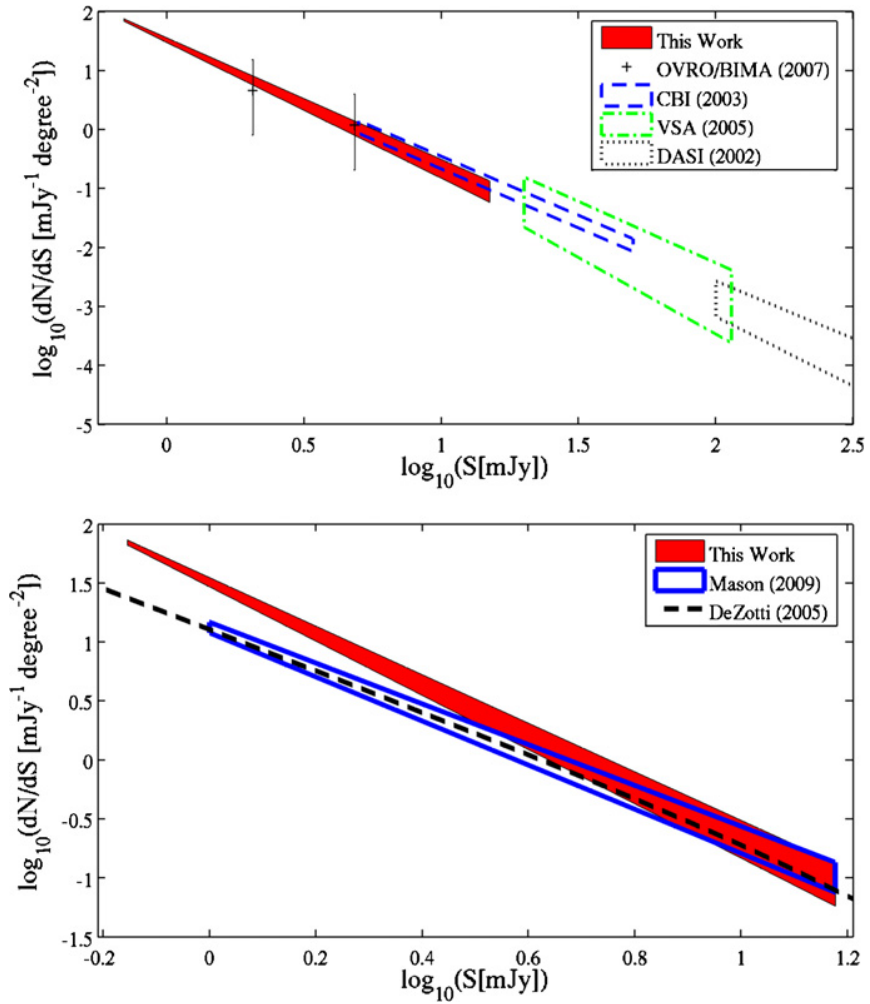

Figure 4. Top: measurements of the $31 \mathrm{GHz} d N / d S$ from this work and prior experimental results from OVRO/BIMA (Coble et al. 2007), CBI (Mason et al 2003), the VSA (Cleary et al. 2005), and DASI (Kovac et al. 2002). Bottom: comparison of the SZA $d N / d S$ to projections from lower frequencies by de Zotti et al. (2005) and Mason et al. (2009).

(A color version of this figure is available in the online journal.)

distributions by properly accounting for the selection thresholds at each frequency. (Kellermann 1964). For example, we can find particular choices of intrinsic spectral index distribution that reproduce both distributions shown in Figure 3 (though in general we have no reason to expect a single distribution to describe both the $1.4 / 31$ and $5 / 31 \mathrm{GHz}$ spectral indices). We therefore refrain from drawing any conclusions about frequency evolution in spectral indices from these data.

\section{DISCUSSION}

In Figure 4, we plot the SZA $d N / d S$ measurement over the range $0.7-15 \mathrm{mJy}$. In the top panel of the figure, we show results from $31 \mathrm{GHz}$ selected sources reported by OVRO/BIMA (Coble et al. 2007), along with results from $31 \mathrm{GHz}$ source studies at higher flux levels, namely, CBI (Mason et al. 2003), DASI (Kovac et al. 2002), and the VSA (Cleary et al. 2005). In the bottom panel, we plot the projection from de Zotti et al. (2005), and from Mason et al. (2009), based on targeted $31 \mathrm{GHz}$ followup of a $1.4 \mathrm{GHz}$ selected sample of sources from the NVSS catalog.

\subsection{Comparison with Cluster Source Measurements}

The only prior measurement of $31 \mathrm{GHz}$ selected sources complete to a comparable flux level is the Coble et al. (2007) sample of $\sim 100$ sources brighter than $1 \mathrm{mJy}$, found serendipitously in targeted observations of massive clusters of galaxies. Since the vast majority of these sources lie within the inner regions of clusters, these measurements permit a direct comparison of the cluster population with the $31 \mathrm{GHz}$ field-source population (this work). By integrating the field-source count from the SZA (Equation (12)) and the cluster source count from Coble et al. $\left(2007 ; N(>S)=\left(709_{-220}^{+580}\right) \mathrm{deg}^{-2} \times\left(S_{\mathrm{mJy}}\right)^{-0.67 \pm 0.037}\right)$ over the flux range $0.7-15 \mathrm{mJy}$, we calculate the overdensity of radio sources within the central arcminute of clusters to be a factor $6.8 \pm 4.4$, consistent with their estimate.

\subsection{Comparison with Field-source Measurements}

From the top panel of Figure 4, we note the general agreement between the SZA result and prior $31 \mathrm{GHz}$ measurements, at the higher completeness levels of those experiments ( $~ \geq 4 \mathrm{mJy}$ ). Agreement with the field-source count of Coble et al. (2007) is likewise good, although their sample in non-cluster fields consists of only four sources. From the bottom panel of Figure 4, we see that the SZA result is also consistent with the projections de Zotti et al. (2005) and Mason et al. (2009, hereafter M09) for fluxes greater than $\sim 4 \mathrm{mJy}$. At low flux, however, they deviate significantly.

The M09 projection is from a study of 3165 sources selected from the NVSS catalog at $1.4 \mathrm{GHz}$ and reobserved at the same central observing frequency as the SZA. Adopting the 1.4 GHz source distribution of Hopkins et al. (2003), and an intrinsic $1.4 / 31 \mathrm{GHz}$ spectral index distribution inferred from their own observations, they estimate the integrated $31 \mathrm{GHz}$ source count over the same flux range as the SZA results to be $N(>S)=(16.7 \pm 1.7) \mathrm{deg}^{-2} \times\left(S_{\mathrm{mJy}}\right)^{-0.80 \pm 0.07}$. This is inconsistent with the count we determine directly at $31 \mathrm{GHz}$ (Section 5.2.3) in both the normalization and the power-law index, as shown in the bottom panel of Figure 4.

As a consistency check, we generate simulated source populations under the set of assumptions outlined in M09, apply the source extraction algorithm detailed in Section 4.1 to the simulated data, and apply the formalism of Section 5.2 to calculate the expected $N(>S)$ at $31 \mathrm{GHz}$. In particular, we generate a list of $1.4 \mathrm{GHz}$ source fluxes over the range $25 \mu \mathrm{Jy}-1 \mathrm{Jy}$ using the source count of Hopkins et al. (2003), and assigning spectral indices according to M09 to extrapolate these fluxes to $31 \mathrm{GHz}$. Sources are then assigned random locations on a noise map identical to the actual SZA survey coverage, with appropriate Gaussian noise added to the $31 \mathrm{GHz}$ fluxes. We select only sources which would have been detected by the SZA, i.e., with $\mathrm{S} / \mathrm{N}$ greater than 5 , and calculate the integrated source count. This procedure is repeated for 100 realizations of source populations, resulting in $N(>S)=\left(16.1_{-2.9}^{+3.3}\right) \mathrm{deg}^{-2} \times\left(S_{\mathrm{mJy}}\right)^{-0.86 \pm 0.18}$. This result is consistent with the prediction of M09, and demonstrates that our methodology is robust to the experimental details of the SZA survey, or differences in source selection between the two measurements.

We have also investigated whether this discrepancy can be due to differences in resolution between the SZA and the VLA in its NVSS configuration. However, of the 209 sources detected with the SZA, we note that in only seven cases are multiple sources close enough to appear as a single source in the NVSS beam. Treating these cases as single sources, we obtain $N(>S)=(26.1 \pm 2.3) \mathrm{deg}^{-2} \times\left(S_{\mathrm{mJy}}\right)^{-1.19 \pm 0.13}$, an insignificant change from our nominal result (Equation (12)).

To reconcile the two measurements, we postulate a change in the $1.4 / 31 \mathrm{GHz}$ spectral index distribution for sources below the $1.4 \mathrm{GHz}$ flux limit of the M09 study. Although M09 find no significant difference between the spectral index distributions of $1.4 \mathrm{GHz}$ sources with flux $>10 \mathrm{mJy}$ and sources with flux $<10 \mathrm{mJy}$, we note that their analysis is necessarily limited to sources brighter than the NVSS completeness limit 
of $3.5 \mathrm{mJy}$. Sources below this limit, however, constitute a large fraction of the $1.4 \mathrm{GHz}$ source population used to predict the $31 \mathrm{GHz} d N / d S$, and small changes in the assumed spectral index distribution of this population can have a significant impact on the source population at $31 \mathrm{GHz}$. We note also that the SZA data suggest this hypothesis ab initio; while the M09 distribution predicts that fewer than $10 \%$ of sources detected by the SZA at $31 \mathrm{GHz}$ would lack $1.4 \mathrm{GHz}$ counterparts $>3.5 \mathrm{mJy}$, fully $25 \%$ of SZA sources lack counterparts in the NVSS catalog.

To test this hypothesis, we repeat the simulations described above, using the M09 spectral index distribution for sources above the NVSS completeness limit ( $3.5 \mathrm{mJy})$, and a separate spectral index distribution for sources with $1.4 \mathrm{GHz}$ fluxes below $3.5 \mathrm{mJy}$. For the dim sources, we assume a distribution whose shape is identical to that of M09, but shifted with respect to it. A good match to our data can be obtained by shifting the M09 spectral distribution by 0.35 to more inverted spectra, resulting in a predicted $N(>S)=(25.5 \pm 2.9) \mathrm{deg}^{-2} \times\left(S_{\mathrm{mJy}}\right)^{-1.06 \pm 0.14}$. This distribution has a peak spectral index of $\sim 0.7$, and predicts that $\sim 7 \%$ of dim sources are inverted between 1.4 and $31 \mathrm{GHz}$. This ad hoc model also accounts for the observed fraction of SZA sources that fall below the NVSS detection threshold. In addition, this model provides a better fit than the singlecomponent model to the observed $1.4 / 31 \mathrm{GHz}$ spectral index distribution shown in Figure 3. Note that while this ansatz agrees well with our data, a physically motivated model of faint-source spectra is likely to be much more complex.

Follow-up studies of faint $1.4 \mathrm{GHz}$ sources at higher frequency have indeed found evidence for a flattening of the spectral index distribution near the mJy level. Prandoni et al. (2006) find that for sources whose $1.4 \mathrm{GHz}$ flux is greater than $4 \mathrm{mJy}$, no sources are inverted between 1.4 and $5 \mathrm{GHz}$, but that for sources dimmer than $4 \mathrm{mJy}$ at $1.4 \mathrm{GHz}$, roughly $10 \%$ of sources are inverted, comparable to the fraction of inverted sources we find between 1.4 and $31 \mathrm{GHz}$. In addition, the study of Donnelly et al. (1987) concludes that the median $1.4 / 5 \mathrm{GHz}$ spectral index is roughly 0.75 down to a $1.4 \mathrm{GHz}$ flux of $0.25 \mathrm{mJy}$, and that the fraction of flat spectrum sources increases from $22 \%$ at $>0.5 \mathrm{mJy}$ to $41 \%$ in the $0.25-0.5 \mathrm{mJy}$ range.

\section{CONCLUSIONS}

We present a sample of $31 \mathrm{GHz}$ selected sources from a $7.7 \mathrm{deg}^{2}$ survey obtained with the SZA. We identify 209 sources at $>5 \sigma$ detection significance, ranging in flux from $0.7-204 \mathrm{mJy}$. A maximum-likelihood determination of the integrated source count results in $N(>S)=(27.2 \pm 2.5) \mathrm{deg}^{-2} \times\left(S_{\mathrm{mJy}}\right)^{-1.18 \pm 0.12}$ in the flux range $0.7-15 \mathrm{mJy}$. Comparison with a measurement of $31 \mathrm{GHz}$ sources toward massive galaxy clusters leads to an overdensity of $6.8 \pm 4.4$, for sources within the central arcminute of massive clusters, relative to field sources.

Of the existing source samples selected at $31 \mathrm{GHz}$, the SZA sample represents the only unbiased study of sources valid to $<1 \mathrm{mJy}$. This sample will therefore be useful in refining source models that are currently constrained by measurements at much higher flux. Such models are important for the accurate in- ference of cosmological information from the current generation of centimeter-wave CMB anisotropy and SZ cluster survey measurements, whose primary contaminant is compact radio sources.

We thank John Cartwright, Ben Reddall, and Marcus Runyan for their significant contributions to the construction and commissioning of the SZA instrument. We thank the staff of the Owens Valley Radio Observatory and CARMA for their outstanding support. We thank Jonathan Sievers and Brian Mason for helpful discussions, Bryan Butler, Claire Chandler, Gustaaf van Moorsel, and Meri Stanley for their assistance with EVLA mosaicking observations, and Bryan Butler and Mark Gurwell for providing the Mars model to which the SZA data are calibrated. We gratefully acknowledge the James S. McDonnell Foundation, the National Science Foundation and the University of Chicago for funding to construct the SZA. The operation of the SZA is supported by NSF Division of Astronomical Sciences through grant AST-0604982. Partial support is provided by NSF Physics Frontier Center grant PHY-0114422 to the Kavli Institute of Cosmological Physics at the University of Chicago, and by NSF grants AST-0507545 and AST-05-07161 to Columbia University. A.M. acknowledges support from a Sloan Fellowship, and S.M. from an NSF Astronomy and Astrophysics Fellowship, and C.G., S.M., and M.S. from NSF Graduate Research Fellowships.

Facilities: SZA, VLA

\section{REFERENCES}

Bennett, C. L., et al. 2003, ApJS, 148, 97

Cleary, K. A., et al. 2005, MNRAS, 360, 340

Clegg, P. E. 1980, Phys. Scr., 21, 678

Coble, K., et al. 2007, AJ, 134, 897

Condon, J. J., Cotton, W. D., Greisen, E. W., Yin, Q. F., Perley, R. A., Taylor, G. B., \& Broderick, J. J. 1998, AJ, 115, 1693

Crawford, D. F., Jauncey, D. L., \& Murdoch, H. S. 1970, ApJ, 162, 405

de Zotti, G., Ricci, R., Mesa, D., Silva, L., Mazzotta, P., Toffolatti, L., \& González-Nuevo, J. 2005, A\&A, 431, 893

Donnelly, R. H., Partridge, R. B., \& Windhorst, R. A. 1987, ApJ, 321, 94

Hopkins, A. M., Afonso, J., Chan, B., Cram, L. E., Georgakakis, A., \& Mobasher, B. 2003, AJ, 125, 465

Kellermann, K. I. 1964, ApJ, 140, 969

Kovac, J. M., Leitch, E. M., Pryke, C., Carlstrom, J. E., Halverson, N. W., \& Holzapfel, W. L. 2002, Nature, 420, 772

Mason, B. S., Weintraub, L., Sievers, J., Bond, J. R., Myers, S. T., Pearson, T. J., Readhead, A. C. S., \& Shepherd, M. C. 2009, ApJ, 704, 1433 (M09)

Mason, B. S., et al. 2003, ApJ, 591, 540

Massardi, M., et al. 2008, MNRAS, 384, 775

Muchovej, S., et al. 2007, ApJ, 663, 708

Murdoch, H. S., Crawford, D. F., \& Jauncey, D. L. 1973, ApJ, 183, 1

Murphy, T., et al. 2010, MNRAS, 402, 2403

Pearson, T. J., et al. 2003, ApJ, 591, 556

Prandoni, I., Parma, P., Wieringa, M. H., de Ruiter, H. R., Gregorini, L., Mignano, A., Vettolani, G., \& Ekers, R. D. 2006, A\&A, 457, 517

Rudy, D. J., Muhleman, D. O., Berge, G. L., Jakosky, B. M., \& Christensen, P. R. 1987, Icarus, 71, 159

Sharp, M. K., et al. 2010, ApJ, 713, 82

Waldram, E. M., Pooley, G. G., Grainge, K. J. B., Jones, M. E., Saunders, R. D. E., Scott, P. F., \& Taylor, A. C. 2003, MNRAS, 342, 915

White, R. L., Becker, R. H., Helfand, D. J., \& Gregg, M. D. 1997, ApJ, 475, 479 